\title{
Symposium report: breast cancer in India-trends, environmental exposures and clinical implications
}

\author{
Jasmine A. McDonald ${ }^{1,2}$ (]) $\cdot$ Roshni Rao $^{2,3} \cdot$ Marley Gibbons $^{1} \cdot$ Rajiv Janardhanan ${ }^{4} \cdot$ Surinder Jaswal $^{5}$. \\ Ravi Mehrotra ${ }^{6} \cdot$ Manoj Pandey $^{7} \cdot$ Venkatraman Radhakrishnan $^{8} \cdot$ Pooja Ramakant $^{9} \cdot$ Nandini Verma $^{10,11}$. \\ Mary Beth Terry ${ }^{1,2}$
}

Received: 23 November 2020 / Accepted: 29 March 2021 / Published online: 28 April 2021

(c) The Author(s) 2021

\begin{abstract}
Purpose Incidence of breast cancer (BC), particularly in young women, are rising in India. Without population-based mammography screening, rising rates cannot be attributed to screening. Investigations are needed to understand the potential drivers of this trend.

Methods An international team of experts convened to discuss the trends, environmental exposures, and clinical implications associated with BC in India and outlined recommendations for its management.

Results Panels were structured across three major BC themes ( $n=10$ presentations). The symposium concluded with a semistructured Think Tank designed to elicit short-term and long-term goals that could address the challenges of BC in India. Conclusion There was consensus that the prevalence of late-stage BC and the high BC mortality rates are associated with the practice of detection, which is primarily through clinical and self-breast exams, as opposed to mammography. TripleNegative BC (TNBC) was extensively discussed, including TNBC etiology and potential risk factors, the limited treatment options, and if reported TNBC rates are supported by rigorous scientific evidence. The Think Tank session yielded longterm and short-term goals to further BC reduction in India and included more regional etiological studies on environmental exposures using existing India-based cohorts and case-control studies, standardization for molecular subtyping of BC cases, and improving the public's awareness of breast health.
\end{abstract}

Keywords India $\cdot$ Breast cancer $\cdot$ Triple-negative breast cancer $\cdot$ Risk factors $\cdot$ Incidence trends

Jasmine A. McDonald

jam2319@cumc.columbia.edu

1 Department of Epidemiology, Mailman School of Public Health, Columbia University Irving Medical Center, 722 West 168th St, New York, NY 10032, USA

2 Herbert Irving Comprehensive Cancer Center, Columbia University Irving Medical Center, New York, NY, USA

3 Division of Breast, Melanoma and Soft Tissue Surgery, Columbia University Vagelos College of Physicians and Surgeons, Columbia University Irving Medical Center, New York, NY, USA

4 Laboratory of Disease Dynamics \& Molecular Epidemiology, Amity Institute of Public Health, Amity University, Uttar Pradesh, India

5 School of Research Methodology, Centre for Health and Mental Health, School of Social Work, Tata Institute of Social Sciences, Mumbai, Maharashtra, India
6 Indian Council of Medical Research (ICMR) - India Cancer Research Consortium, New Delhi, India

7 Institute of Medical Sciences, Department of Surgical Oncology, Banaras Hindu University, Varanasi, Uttar Pradesh, India

8 Cancer Institute (W.I.A), Chennai, India

9 King Georges Medical University, Lucknow, Uttar Pradesh, India

10 TNBC Precision Medicine Research Laboratory, Advanced Centre for Treatment, Research, and Education in Cancer, Tata Memorial Center, Khargar, Navi Mumbai, Maharashtra, India

11 Homi Bhabha National Institute, Training School Complex, Anushakti Nagar, Mumbai, Maharashtra, India 


\section{Abbreviations \\ APC Annual percent change \\ BC Breast cancer \\ BRCA BReast CAncer gene \\ DALY Disability-adjusted life years \\ EDC Endocrine disrupting chemicals \\ PAH Polycyclic aromatic hydrocarbons \\ TNBC Triple-negative breast cancer \\ US United States}

\section{Introduction}

In India, breast cancer (BC) has emerged as the second most common type of malignancy within the past 25 years. In terms of disability-adjusted life years (DALYs), where BC used to be less prevalent than the more prevalent stomach, cervical, and leukemia diagnoses, according to data accumulated up until 2016, BC is surpassed only by cervical cancer [1]. Moreover, India has one of the highest rates of the most aggressive subtype of $\mathrm{BC}$ referred to as TripleNegative Breast Cancer (TNBC) [2,3]. The rates of TNBC in India are almost double that of the United States (US) with estimates as high as $28 \%$ to one-third of all BC in India compared to $12-15 \%$ in the US [4]. Characterized by the lack of estrogen, progesterone, and HER2Neu receptors, TNBC lacks treatment options for targeted therapy and has a higher fatality rate as compared to other BC subtypes which tends to occur in younger patients (women under age 50) and women of African descent [5].

With the goal of employing collaborative efforts at reducing $\mathrm{BC}$ incidence and mortality in India, a group of cancer experts and key stakeholders were invited to the Breast Cancer in India: Trends, Environmental Exposures and Clinical Implications symposium held at the Columbia Global Centers I Mumbai on 4 December 2019 (see Fig. 1). The three objectives for this meeting were as follows: (1) gather cancer experts and key stakeholders to discuss the challenges and research opportunities to reduce BC in India, (2) consider etiological and potential exposures that may contribute to the rates of early-onset $\mathrm{BC}$, and (3) develop short- and longterm collaborative research and training goals for $\mathrm{BC}$ risk reduction.

In this report, we summarize the interdisciplinary discussions from experts across relevant fields that led to the groups' research, policy and programming, and advocacy recommendations that are needed to address BC in India, particularly for women under 50 years.

\section{Breast cancer-clinical diagnoses, prognoses, and outcomes}

Panelists focused on identifying temporal trends in BC, delineating actual rates of TNBC, and discussing current obstacles to BC care in India.

\section{Younger age}

There was general agreement that BC in India is more often seen in a younger population when compared to Western cohorts. Data from Banaras Hindu University (BHU) reveal a mean age of 51, and data from the Sanjay Gandhi Postgraduate Institute of Medical Sciences (SGPGIMS) in Lucknow, India reports a mean age of 49.7 years. Data from the Indian Council of Medical Research between 1982 and 2005 observed an annual percent change (APC) as high as $4.2 \%$ in Nagpur among women age 15-34 [6]. The group discussed whether the younger age was driven by higher age-specific incidence rates in India or related to biases based on clinical presentation and who is treated and can travel to cancer hospitals may be related to younger ages.

\section{Higher stage}

Another significant issue is the stage of presentation. A cohort of 3,473 patients from SGPGIMS demonstrated an $11 \%$ incidence of Stage IV disease at presentation, and although this did decrease over time from 1990 when the incidence of Stage IV disease was $20 \%$, this is significantly higher than the $6 \%$ incidence observed in the US [7]. The group at BHU reported a 35\% incidence of Stage IV BC at initial presentation. However, given this is not populationbased data but hospital-based data from an institution with tertiary care center status, "referral bias" is an issue.

There was no consensus on what screening modality would best serve the Indian population to downshift the stage of presentation. Given the known limitations of mammography in dense breast tissue and in young women, attendees agreed that population-based mammography would not be optimal as a population-based approach for screening particularly given the earlier age of onset in Indian women [8]. The value of ultrasound screening requires further investigation and there are concerns about overwhelming the health care infrastructure with false positives. It is unclear whether clinical breast examination alone is sufficient, and the challenge would be determining who should perform this and what training level is required to maximize benefit. Another key recommendation would be to identify optimal screening avenues (and community initiatives with the help of local primary care workers) for the Indian population. 
Fig. 1 .

Breast Cancer in India: Trends, Environmental Exposures and Clinical Implications

December 3-4, 2019

Columbia Global Center | Mumbai

Hosted by Drs. Mary Beth Terry, Jasmine McDonald, and Ravina Aggarwal

SYMPOSIUM AGENDA

Wednesday, 4 December 2019

10:00-10:15 Registration, tea/coffee, small bites

10:15-10:55 Session 1: Introductions \& Objectives

Welcome to Symposium

Ravina Aggarwal, Columbia Global Centers | Mumbai

The Yusuf K. Hamied Faculty Fellowship Experience

Surinder Jaswal, Tata Institute of Social Sciences, Mumbai

Overview of Symposium's Objectives and Goals

Mary Beth Terry and Jasmine McDonald, CUIMC

11:00-11:45 Session 2: Breast Cancer-Clinical Diagnoses, Prognoses, and Outcomes

Discussant: Roshni Rao, CUIMC

Panelists:

Gaurav Agarwal, Sanjay Gandhi Post Graduate Institute of Medical Sciences, Lucknow

Manoj Pandey, Institute of Medical Sciences, Banaras Hindu University, Varanasi

11:45-12:00 Discussion

12:05-12:50 Session 3: Molecular and Pathological Differences in Breast Cancer

Discussant: Jasmine McDonald, CUIMC

Panelists:

Venkatraman Radhakrishnan, Cancer Institute W.I.A, Chennai

Pooja Ramakant, King George's Medical Institute, Lucknow

Tanuja Shet, Tata Memorial Center, Mumbai

12:50-13:05 Discussion

13:05-14:05 Lunch

14:10-15:15 Session 4: What Are Our Current Gaps in Knowledge?

Discussant: Dr. Mary Beth Terry, CUIMC

Panelists:

Ravi Mehrotra, IMCR - Indian Cancer Research Consortium, New Delhi

Rajesh Dikshit, Centre for Cancer Epidemiology, Tata Memorial, Mumbai

15:15-15:25 Discussion

15:25-15:40 Coffee \& Tea Break

15:40-17:20 Session 5: Interactive Think Tank Series

Think Tank Introduction

Moderators: Drs. Terry and McDonald, CUIMC

Breakout Round Tables: Brainstorming about Potential Study Designs

Report back \& group action, role assignments

17:20-17:40 Closing Remarks: Next steps and possible sources of funding Jasmine McDonald \& Mary Beth Terry, CUIMC

\section{TNBC}

Single-institution data support higher rates of TNBC in the Indian population. The BHU group reports a $32 \%$ $(n=113 / 349)$ rate of TNBC and SGPGIMS data indicate a 29\% $(n=1,042 / 3,473)$ incidence of TNBC. These are both higher than what is observed in Western populations, although some research indicates the oncologic outcomes in the Indian population may not be influenced as heavily by hormone status [9]. This finding may be related 
to the overall lower survival from $\mathrm{BC}$ when it presents with advanced disease. The recommendation from the group includes the standardization of pathologic reporting and analysis (outlined in the following sections), as well as consideration for a national TNBC registry to prospectively collect national level data that can also underline the incidence of $\mathrm{BC}$ by state to understand regional epidemiology.

\section{Molecular and pathological differences in breast cancer}

\section{Debate: TNBC rates}

Are TNBC rates in India rising? As noted above, India-based TNBC data are limited by the lack of population-based rates $[4,10]$; nonetheless, some experts suggest that TNBC incidences are rising. A recent meta-analysis across 17 crosssectional studies found a 31\% prevalence of TNBC in India with study heterogeneity not explained by available studylevel characteristics (i.e., age, menopausal status, grade, tumor size). This high prevalence is observed in women of African descent, with population-based studies observing between 21 and $46 \%$ among Black women in the United States and $20 \%$ to $82 \%$ in African women [11]. Additional factors for TNBC include younger age, BRCA1 mutation status, higher body mass index, and a host of reproductive factors, including earlier age at menarche, younger age at first full-term birth, higher parity, and shorter duration of breastfeeding. How these risk factors distribute across Indian women with TNBC is of interest.

Several suggested differences in TNBC rates were driven by the distribution of tumors observed in India compared to countries that have population-based screening and therefore a larger proportion of smaller tumors [12]. TNBC rates may also not be as high as estimates suggest as standards related to core biopsy collection, processing, and receptor assessments are not consistent across India [13]. While some hospitals have the resources to adequately prepare the core biopsy samples and implement standardized ER/ PR and HER2 testing with routine quality checks to ensure standards of assessments are met, some hospitals are lacking these practices or the access to quality molecular testing. This possible faulty testing could result in an over-estimate. However, it is also possible that as these standardizations are put in place, while performance will improve, the proportions of TNBC may remain constant, which would suggest that TNBC rates are not changing.

\section{Improving TNBC classification and risk stratification}

TNBC is a heterogeneous disease where the majority are basal-like subtype, but a TNBC diagnosis requires confirmation by gene expression signatures and immunohistochemistry analysis [12], which experts agreed could present significant methodological challenges. Challenges in subtyping lead to challenges in identifying novel treatments and more effectively targeted drugs [14]. For example, attempts to reproduce certain TNBC subtypes have been inconsistent and therefore researchers are unable to validate gene signatures predictive of disease-free survival [14]. Overall, more research is needed on these aggressive tumors, including examination of precursor lesions for TNBC and the exploration of possible causal associations between infectious agents (e.g., EBV, microbiome, etc.) and TNBC. Of universal concern was that the majority of $\mathrm{BC}$ cases in India are late stage which results in poorer BC prognoses. However, with limited effective treatment options for TNBC, the drugs that are available do little to improve cancer prognoses [15, 16]. To improve prognoses, women need to be observed at earlier stages. A step toward this would be implementing risk prediction modeling. For example, some of the genes that are associated with TNBC are also genes that might be able to be screened for across different high-risk populations [17].

\section{What are our current gaps in knowledge?}

In addition to greater standardization in molecular subtyping, there is also a pressing need to expand etiological research into what may be the key drivers of the increase in $\mathrm{BC}$ rates. As population-based screening mammography is not utilized in India, increased screening is not an explanation for the rising incidence trends.

\section{Geographic variation: what are the drivers?}

In addition to the increasing trends throughout India, there remains substantial geographic variation in $\mathrm{BC}$ incidence across India from a high of 240.8 DALY rate in Kerala to a low of 58.4 DALY rate in Sikkim [1]. Importantly, not only is there substantial variation in DALY rates across India but also within areas of similar epidemiological transition levels [9]. The great variability across and within regions supports that there is a need to understand the drivers of these differences, including difference in demographic characteristics and access to resources across regions/states, particularly in relation to class and caste and rural, tribal, and urban settings.

There is growing evidence that established risk factors for $\mathrm{BC}$, including genetic variants in $\mathrm{BC}$ susceptibility 
genes, are also important predictors of BC risk in India. For example, in a large case-control study with over 1,600 cases and 1,500 controls conducted in Mumbai India, Dr. Dikshit and colleagues reported that central adiposity (measured by a high waist to hip ratio) was associated with both preand postmenopausal BC [18]. His team also reported that 11 genetic variants from eight genomic regions $(F G F R 2$, 9q31.2, MAP3K, CCND1, ZM1Z1, RAD51L11, ESR1, and UST) were also associated with $\mathrm{BC}$ cases and controls from Mumbai, India, and this study also reported an increased prevalence of a number of estrogen metabolizing genes in cases compared to controls [19]. Of note, although many established BC risk factors, like central adiposity and late age at first pregnancy in rural and urban areas of India, the prevalence of these risk factors vary across rural and urban settings [20].

At the symposium, discussion about the role of early life exposures, including risk factors that may be related to growth and breast development and windows of susceptibility in driving BC trends [21], was supported by recent data presented by Dr. Dikshit showing that women who spent the first 20 years of life in rural settings compared to women who spent their first 20 years of life in urban settings had a lower breast cancer risk (personal communication). There was universal agreement that more etiologic research was needed particularly on environmental exposures of air pollution and polycyclic aromatic hydrocarbons (PAHs), and other environmental pollutants and endocrine disrupting chemicals (EDCs). EDCs interfere with various aspects of metabolism and is also a risk factor for metabolic diseases, such as obesity, which is indeed a risk factor strongly associated with aggressive breast cancer lesions. Participants discussed that sustained human exposure to these chemicals may have a disrupting role in the functioning of the endocrine/hormonal system and metabolic pathways [21, 22] and the need for additional studies to be conducted in India.

\section{Improving existing infrastructure}

The panelists reviewed screening recommendations already established through governmental public health organizations, including (1) clinical breast examination combined with diagnostic ultrasound; (2) awareness and education supplemented with clinical breast examination screening and early detection programs that should bring women in for examination at least once every 3 years; and (3) fine needle aspiration cytological tests or core needle biopsy with appropriate follow-up services should be made available to provide patient access to prompt diagnosis and treatment [23]. Dr. Ravi Mehrotra, Director of the India Cancer Research Consortium, outlined existing mechanisms and coverage of $\mathrm{BC}$ screening through integration and training of health care providers from many different levels of the society, including village health care workers, nurses, dentists, gynecologists, surgeons, and radiologists [24].

Thus, the major gaps in knowledge called for (1) more research examining environmental exposures and additional studies of early life exposures; (2) implementation of scientific methods to identify ways to increase clinical breast exams as well as increasing awareness of $\mathrm{BC}$ risk and $\mathrm{BC}$ risk factors; and (3) evaluation of screening strategies to reduce the burden of late-stage diagnoses.

\section{Suggestions and recommendations}

The Think Tank portion of the symposium was a facilitatorled semi-structured brainstorming intended to discuss some of the short-term and long-term goals that could address BC in India. Throughout the discussion, attendees referred to the Pulse Polio and National AIDS Control Programme (NACP) as successful campaigns we could learn from and possibly build upon in order to implement elements of the recommendations. During the session, attendees gathered in smaller preassigned groups to develop recommendations for short- and long-term research strategies for primary prevention and for short- and long-term solutions focused on clinical outcomes. The "best picks" were reported to the group followed by brief discussion. In closing, each attendee shared personal thoughts on what would be the most pressing strategy for addressing the burden of early-onset BC in India. The recommendations fell into three actionable elements: Research, Policy/Programming, and Advocacy (Table 1).

\section{Summary of recommendations (Table 1)}

Recommendations included de novo efforts as well as building on the success of existing infrastructure or efforts [25]. One of the broadest reaching recommendations aimed to reduce stigma around what it means to be a female in Indian society. Breast cancer risk reduction and improved early detection and screening demands a better understanding by health care providers and Indian Public Health officials of the barriers women face when considering self-breast examination. One should consider a woman's comfortability in performing a self-breast examination as well as the more nuanced comfortability of asking questions about the risk of breast cancer or even seeking treatment. Therefore, the recommendation is a multi-level effort to understand and address the core cultural barriers to body awareness that provides programming and resources for improved $\mathrm{BC}$ prevention, detection, and treatment at all levels. 


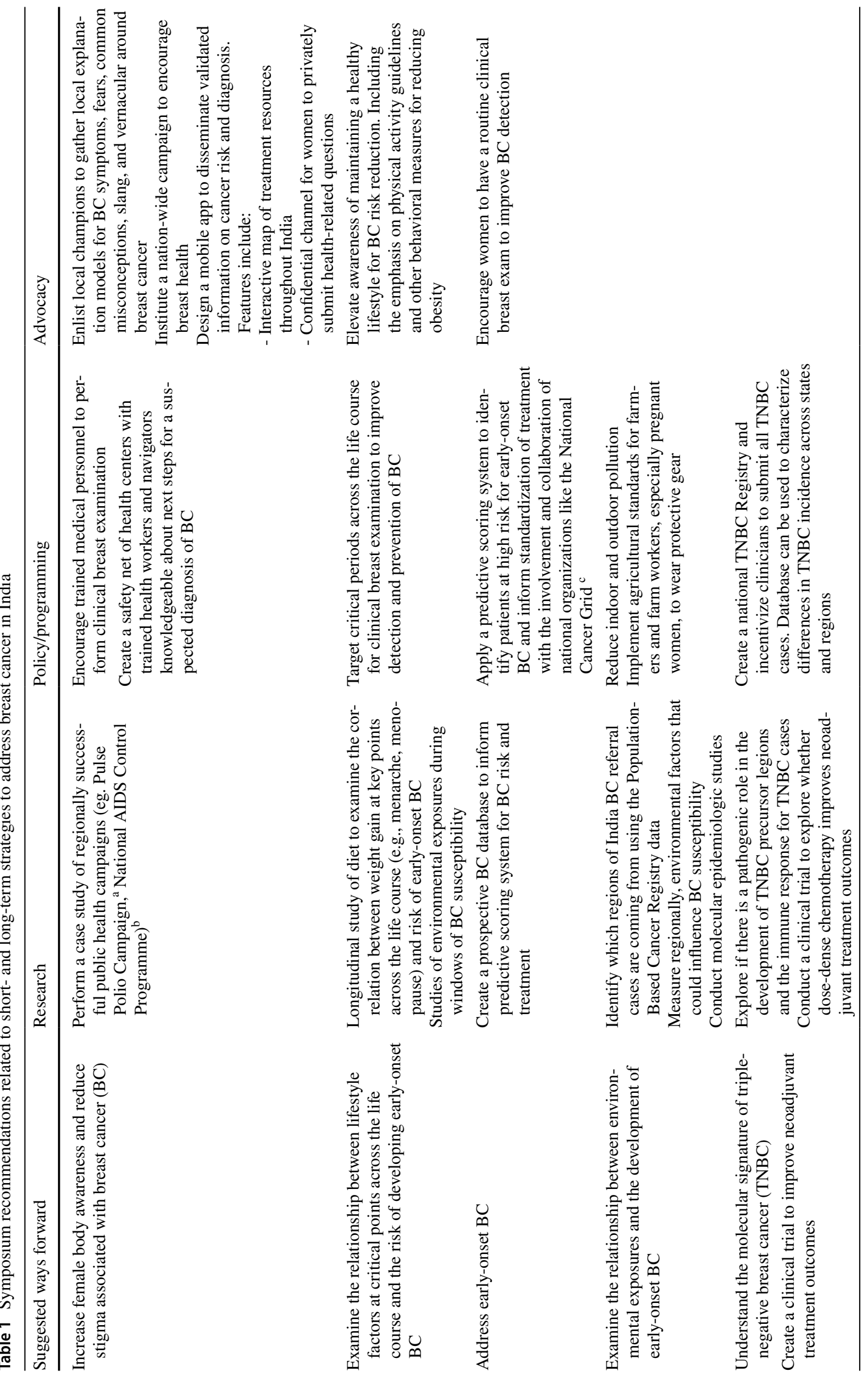




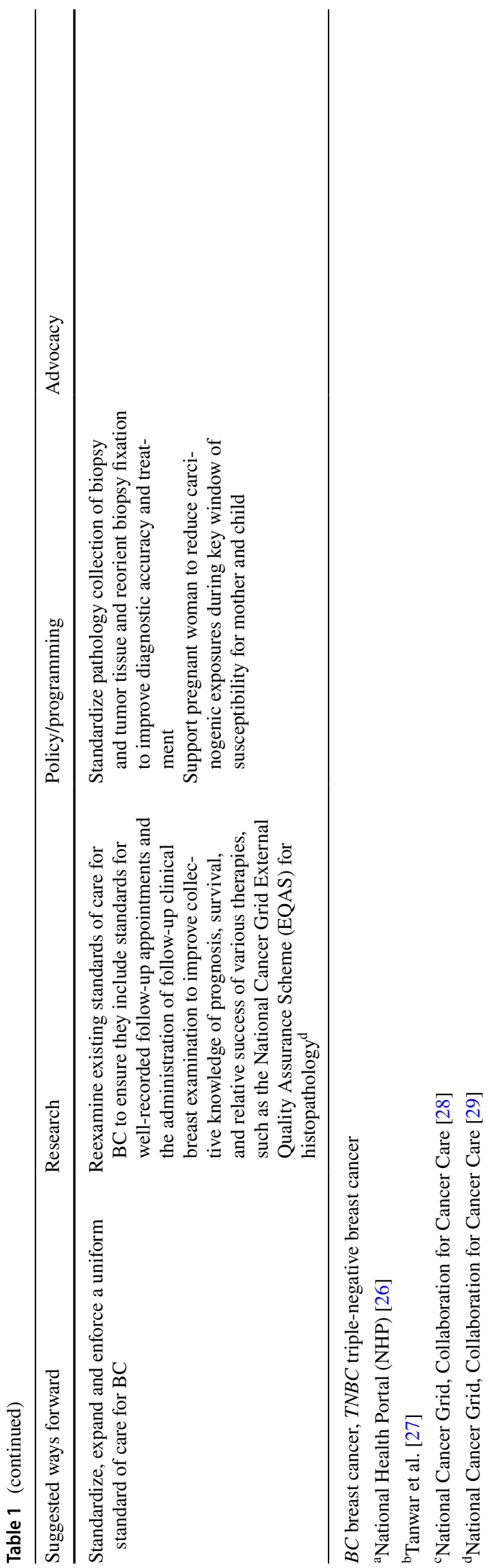

\section{Standardization as a key to moving forward}

Another major summary of our discussions included the need to address the disagreement within the medical community about the nature and severity of the problem itself. While attendees agreed that early-onset BC is a major concern, there was much disagreement about accuracy (e.g., have rates increased or has reporting just improved?). This issue underscored the need for improved standardization of molecular subtyping across clinical subtyping in India. Increased standardization and monitoring for data quality as well as pooling of data across regions will help improve monitoring of treatment outcomes. There was agreement that there needs to be a greater implementation of a national uniform standard of care.

\section{Conclusion}

All experts agreed that greater awareness of women's health and $\mathrm{BC}$ is needed across India as well as the fact that more needs to be done to understand hereditary breast cancer in India, as a unique BRCA variant has been described in the Indian population [6]. The higher rate of triple-negative $\mathrm{BC}$ observed in India in comparison to other countries may be partially explained by the detection methods in India, but more research and a National Breast Cancer Registry is needed. A call for improved standardization of molecular subtyping across clinical centers in India as well as etiological studies of environmental exposures and $\mathrm{BC}$ within existing cohorts and case-control studies will be essential to understand the drivers of early age of onset and the etiology behind increasing $\mathrm{BC}$ incidence rates in India.

Acknowledgments The authors thank the Yusuf K. Hamied Fellowship, the Columbia Global Center I Mumbai, Dr. Kavita Sivaramakrishnan, and Mrs. Caitlin Hawke for the support that made this symposium possible. They thank all those who participated in the symposium and offered their insights and contributed to the discussion.

Funding This work was supported by the Yusuf K. Hamied Fellowship Award and by funding from the National Cancer Institute grant K01 CA186943 and the Breast Cancer Research Foundation.

\section{Declarations}

Conflict of interest All the authors declare that they have no conflict of interest.

Ethical approval This study does not contain any studies with human participants or animals performed by any of the authors.

Open Access This article is licensed under a Creative Commons Attribution 4.0 International License, which permits use, sharing, adaptation, distribution and reproduction in any medium or format, as long as you give appropriate credit to the original author(s) and the source, 
provide a link to the Creative Commons licence, and indicate if changes were made. The images or other third party material in this article are included in the article's Creative Commons licence, unless indicated otherwise in a credit line to the material. If material is not included in the article's Creative Commons licence and your intended use is not permitted by statutory regulation or exceeds the permitted use, you will need to obtain permission directly from the copyright holder. To view a copy of this licence, visit http://creativecommons.org/licenses/by/4.0/.

\section{References}

1. Dhillon PK et al (2018) The burden of cancers and their variations across the states of India: the global burden of disease study 1990-2016. Lancet Oncol 19(10):1289-1306. https://doi.org/10. 1016/S1470-2045(18)30447-9

2. Torre LA, Siegel RL, Ward EM, Jemal A (2016) Global cancer incidence and mortality rates and trends-an update. Cancer Epidemiol Biomark Prev 25(1):16-27. https://doi.org/10.1158/10559965.Epi-15-0578

3. World Health Organization (2012) Globocan 2012: estimated cancer incidence, mortality and prevalence worldwide In: 2012. https://publications.iarc.fr/databases/iarc-cancerbases/globocan2012-estimated-cancer-incidence-mortality-and-prevalence-world wide-in-2012-v1.0-2012. Accessed 10 June 2020

4. Thakur KK, Bordoloi D, Kunnumakkara AB (2018) Alarming burden of triple-negative breast cancer in India. Clin Breast Cancer 18(3):E393-E399. https://doi.org/10.1016/J.Clbc.2017.07.013

5. Foulkes WD, Smith IE, Reis-Filho JS (2010) Triple-negative breast cancer. N Engl J Med 363(20):1938-1948. https://doi.org/ 10.1056/Nejmra1001389

6. Malvia S, Sa B, Us D, Saxena S (2017) Epidemiology of breast cancer in indian women. Asia Pac J Clin Oncol 13(4):289-295. https://doi.org/10.1111/Ajco.12661

7. Cronin KA, Lake AJ, Scott S, Sherman RL, Noone AM, Howlader N, Henley SJ, Anderson RN, Firth AU, Ma J, Kohler BA, Jemal A (2018) Annual report to the nation on the status of cancer, part I: National Cancer Statistics. Cancer 124(13):2785-2800. https:// doi.org/10.1002/Cncr.31551

8. Winchester DP (1996) Breast cancer in young women. Surg Clin North Am 76(2):279-287. https://doi.org/10.1016/S00396109(05)70439-4

9. Agarwal G, Nanda G, Lal P, Mishra A, Agarwal A, Agrawal V, Krishnani N (2016) Outcomes of triple-negative breast cancers (Tnbc) compared with non-tnbc: does the survival vary for all stages? World J Surg 40(6):1362-1372. https://doi.org/10.1007/ S00268-016-3422-4

10. Sandhu GS, Erqou S, Patterson H, Mathew A (2016) Prevalence of triple-negative breast cancer in India: systematic review and metaanalysis. J Glob Oncol 2(6):412-421. https://doi.org/10.1200/Jgo. 2016.005397

11. Dietze EC, Sistrunk C, Miranda-Carboni G, O'regan R, Seewaldt VL (2015) Triple-negative breast cancer in African-American Women: disparities versus biology. Nat Rev Cancer 15(4):248254. https://doi.org/10.1038/Nrc3896

12. Sable M, Pai TD, Shet T, Patil A, Dhanavade S, Desai SB (2016) Triple-negative breast cancer: a comprehensive study of clinical, histomorphological, and immunohistochemical features in Indian patients. Int J Surg Pathol 25(3):230-237. https://doi.org/10.1177/ 1066896916667815

13. Rangarajan B, Shet T, Wadasadawala T, Ns N, Rm S, Ss H, Bajpai J (2016) Breast cancer: an overview of published Indian data. South Asian J Cancer 5(3):86-92. https://doi.org/10.4103/2278330x.187561
14. Garrido-Castro AC, Lin NU, Polyak K (2019) Insights into molecular classifications of triple-negative breast cancer: improving patient selection for treatment. Cancer Discov 9(2):176-198. https://doi.org/10.1158/2159-8290.Cd-18-1177

15. Bianchini G, Jm B, Ia M, Me S, Gianni L (2016) Triple-negative breast cancer: challenges and opportunities of a heterogeneous disease. Nat Rev Clin Oncol 13(11):674-690. https://doi.org/10. 1038/Nrclinonc. 2016.66

16. Nagini S (2017) Breast cancer: current molecular therapeutic targets and new players. Anticancer Agents Med Chem 17(2):152163. https://doi.org/10.2174/1871520616666160502122724

17. Shimelis H, Laduca H, Hu C, Hart SN, Na J, Thomas A, Akinhanmi M, Rm M, Brauch H, Cox A, Eccles DM, Ewart-Toland A, Fasching PA, Fostira F, Garber J, Godwin AK, Konstantopoulou I, Nevanlinna H, Sharma P, Yannoukakos D, Yao S, Feng BJ, Tippin DB, Lilyquist J, Pesaran T, Goldgar DE, Polley EC, Dolinsky JS, Couch FJ (2018) Triple-negative breast cancer risk genes identified by multigene hereditary cancer panel testing. J Natl Cancer Inst 110(8):855-862. https://doi.org/10.1093/Jnci/Djy 106

18. Nagrani R, Mhatre $S$, Rajaraman P, Soerjomataram I, Boffetta $P$, Gupta S, Parmar V, Badwe R, Dikshit R (2016) Central obesity increases risk of breast cancer irrespective of menopausal and hormonal receptor status in women of South Asian ethnicity. Eur J Cancer (Oxford, England: 1990) 66:153-161. https://doi.org/10. 1016/J.Ejca.2016.07.022

19. Nagrani R, Mhatre S, Rajaraman P, Chatterjee N, Mr A, Boffetta P, Brennan P, Badwe R, Gupta S, Dikshit R (2017) Association of genome-wide association Study (Gwas) identified snps and risk of breast cancer in an Indian population. Sci Rep 7:40963. https:// doi.org/10.1038/Srep40963

20. Nagrani R, Mhatre S, Boffetta P, Rajaraman P, Badwe R, Gupta S, Romieu I, Parmar V, Dikshit R (2016) Understanding rural-urban differences in risk factors for breast cancer in an Indian population. Cancer Causes Control CCC 27(2):199-208. https://doi.org/ 10.1007/S10552-015-0697-Y

21. Terry MB, Michels KB, Brody JG, Byrne C, Chen S, Jerry DJ, Malecki KMC, Martin MB, Miller RL, Neuhausen SL, Silk K, Trentham-Dietz A (2019) Environmental exposures during windows of susceptibility for breast cancer: a framework for prevention research. Breast Cancer Res BCR 21(1):96. https://doi.org/ 10.1186/S13058-019-1168-2

22. Janardhanan R (2018) Endocrine disrupting chemical induced "pollution of metabolic pathways": a case of shifting paradigms with implications for vascular diseases. Curr Drug Targets 19(9):1024-1037. https://doi.org/10.2174/138945011966618 0514122244

23. Rajaraman P, Anderson BO, Basu P, Belinson JL, Cruz AD, Dhillon PK, Gupta P, Jawahar TS, Joshi N, Kailash U, Kapambwe S, Katoch VM, Krishnan S, Panda D, Sankaranarayanan R, Selvam JM, Shah KV, Shastri S, Shridhar K, Siddiqi M, Sivaram S, Seth T, Srivastava A, Trimble E, Mehrotra R (2015) Recommendations for screening and early detection of common cancers In India. Lancet Oncol 16(7):E352-361. https://doi.org/10.1016/ S1470-2045(15)00078-9

24. Dhanasekaran K, Babu R, Kumar V, Mehrotra R, Hariprasad R (2019) Capacity building of gynecologists in cancer screening through hybrid training approach. J Cancer Educ. https://doi.org/ 10.1007/S13187-019-01589-0

25. Hariprasad R, Palat G, Mehrotra R, Vallath N (2018) The pep project-synergistic community based action in prevention, early detection and palliative care, to impact the cancer burden In India. Indian J Palliat Care 24:349-354

26. National Health Portal (NHP) (2015) Pulse Polio Programme. NHP CC DC. https://www.nhp.gov.in/pulse-polio-programme_ pg. Accessed 10 June 2020 
27. Tanwar S, Rewari BB, Rao CD, Seguy N (2016) India's HIV programme: successes and challenges. J Virus Erad 2(Suppl 4):15-19

28. National Cancer Grid, Collaboration for Cancer Care (2016). Tata Memorial Hospital. https://tmc.gov.in/ncg/index.php. Accessed 9 June 2020

29. National Cancer Grid, Collaboration for Cancer Care (2016). Tata Memorial Hospital EQAS program. https://tmc.gov.in/ncg/index. php/ncg-eqas1. Accessed 9 June 2020
Publisher's Note Springer Nature remains neutral with regard to jurisdictional claims in published maps and institutional affiliations. 